\title{
MTRR gene variant rs1801394 found in Malaysian patients with neural tube defects
}

\author{
Amelia Cheng Wei Tan ${ }^{1}$, Siti Waheeda Mohd-Zin ${ }^{1}$, Nur'Awatif Ishak ${ }^{1}$, Meow-Keong Thong ${ }^{2}$, \\ Azlina Ahmad-Annuar ${ }^{3}$, Abu Bakar Azizi ${ }^{4}$ \& Noraishah Mydin Abdul-Aziz ${ }^{1 *}$ \\ ${ }^{1}$ Department of Parasitology, Faculty of Medicine, University of Malaya, 50603 Kuala Lumpur, Malaysia. \\ 2 Department of Paediatrics, Faculty of Medicine, University of Malaya, 50603 Kuala Lumpur, Malaysia. \\ ${ }^{3}$ Department of Biomedical Science, Faculty of Medicine, University of Malaya, 50603 Kuala Lumpur, Malaysia. \\ ${ }^{4}$ Neurosurgery Unit, Department of Surgery, Faculty of Medicine, Universiti Kebangsaan Malaysia, 56000 Cheras, \\ Kuala Lumpur, Malaysia.
}

* Correspondence: noisha@ummc.edu.my; Tel.: +6017-2604310

Received: 7 November 2019; Accepted: 19 February 2020; Published: 5 March 2020

Edited by: Jambaldorj Jamiyansuren (Mongolian National University of Medical Sciences, Mongolia)

Reviewed by: Purevsuren Losol (Mongolian National University of Medical Sciences, Mongolia);

Bilegtsaikhan Tsolmon (Mongolian National University of Medical Sciences, Mongolia)

https://doi.org/10.31117/neuroscirn.v3i1.41

\begin{abstract}
Neural tube defects (NTDs) are congenital anomalies resulting from the failure of neural tube closure during embryogenesis. The precise molecular mechanisms underlying this multifactorial disease is poorly understood, although single nucleotide polymorphisms in genes involved in the one-carbon metabolism cycle are believed to contribute towards NTD development. Among them is 5-methyltetrahydrofolate-homocysteine methyltransferase reductase (MTRR). Protein function prediction algorithms (PolyPhen-2, PROVEAN, SIFT, SMARTEnsembl) were employed to evaluate its pathogenicity potential caused by the replacement of isoleucine with methionine. Seven NTD patients and 12 of their parents were recruited for this study. DNA samples were collected through blood or saliva whereby the extracted DNAs were then sent for whole exome sequencing (WES). Zygosity of the variant was confirmed from WES data of each subject and further validated through polymerase chain reaction (PCR) and Sanger sequencing. The results revealed that $57 \%$ of patients and $83 \%$ of parents carried rs 1801394 mutation in their MTRR gene, based on either homozygous (G/G) or heterozygous (A/G) genotypes. Bioinformatics analysis of this missense mutation predicted that this change is damaging to MTRR protein function by 2 of the 3 predictor algorithms and that the change from isoleucine to methionine amino acid affects flavodoxin domain of the protein. This impacts enzyme activity within the one-carbon metabolism pathway, which is linked to the aetiology of NTDs. From population databases, this variant was considered common with a MAF $>0.3$, however, it was not found in the Singapore Genome Variation Project (SGVP), whose population is a closer representation of the Malaysian subjects investigated here. Hence, we explored the prevalence of this variant in other studies and found that its association with NTDs differed across populations worldwide. Finally, we conclude that rs1801394 may be an NTD risk factor in the Malaysian population and should be further investigated as a potential prenatal screening tool.
\end{abstract}

Keywords: Neural tube defects; MTRR; gene polymorphism; rs1801394; Malaysian 
(C2020 by Tan et al. for use and distribution in accord with the Creative Commons Attribution (CC BY-NC 4.0) license (https://creativecommons.org/licenses/by-nc/4.0/), which permits unrestricted non-commercial use, distribution, and reproduction in any medium, provided the original author and source are credited.

\subsection{INTRODUCTION}

Neural tube defects (NTD), encompassing both brain and spinal cord, are congenital anomalies affecting on the average, 0.5 to 10 per 1000 pregnancies globally (Greene \& Copp, 2014). NTDs result from failure of neural tube closure during embryogenesis, with the exposed neuroepithelial cells susceptible to degeneration in utero (Mohd-Zin et al., 2017). Depending on the site of the lesion along the neuraxis, the severity and types of NTD vary, with defects at the brain (anencephaly) being the most severe phenotype and incompatible with postnatal life. As for lesions affecting the spine, also known as spina bifida, lifelong neurological handicap in patients is to be expected.

NTD is a complex disease with multifactorial aetiology, involving both genetic and environmental contributions. Research has been focused on elucidating the mechanisms and causes of neural tube closure failure, with periconceptional folic acid supplementation singled out as a key nutrient recommended by World Health Organization for primary prevention of NTD. As such, NTD cases in countries where fortification of folic acid is mandatory reports of reduction in prevalence (Kondo et al., 2017). While the recommendation of folic acid supplementation for women of child-bearing age periconceptionally has decreased NTD incidence by $70 \%$, the remaining 30\% remained elusive (Blencowe et al., 2010). For this $30 \%$, it is postulated that predisposing genotypes work together with nongenetic factors in modulating neural tube closure (Copp et al., 2015).

In view of folic acid's role in successfully mitigating a large proportion of NTD cases, it logically follows that genes related to folate metabolism are most widely investigated. The most promising candidate gene to date is 5,10-methylenetetrahydrofolate reductase (MTHFR) gene. It is known that MTHFR influences folate status by irreversibly converting 5,10 methylenetetrahydrofolate (5,10-methylene THF) to 5methyltetrahydrofolate (5-methyl THF) and that the common C677T gene variant reduces its enzymatic activity. As a result, carriers of the T allele(s), both homozygous and heterozygous, have elevated homocysteine levels and lowered folate concentrations, contributing towards NTD susceptibility (Kirke et al., 2004). The second polymorphism of this gene, A1298C, is suggested to decrease MTHFR activity through gene-gene interaction with C677T variant (Zhang et al., 2013). However, despite the strong case for C677T as an independent risk factor for NTD, it appears that the prevalence of these MTHFR variants vary across populations and ethnic groups (Zhang et al., 2013; van der Put et al., 1997; Amorim et al., 2007; Del Gobbo et al., 2018), including a negative association in Malaysian Malays (Hayati et al., 2008).

As such, research to probe for single nucleotide polymorphisms (SNP) in other genes in the one-carbon metabolism cycle is currently underway. One of the prime candidates is the 5-methyltetrahydrofolatehomocysteine methyltransferase reductase (MTRR) gene. MTRR gene, encoding for methionine synthase reductase protein, is targeted in light of its chaperone role for 5-methyltetrahydrofolate-homocysteine methyltransferase (MTR) protein, and the key regulatory role it plays in folate metabolism. Located on chromosome 5, a missense mutation in MTRR is believed to impair the functions of methionine synthase, an enzyme linking together folate and methylation cycles in the one-carbon metabolism pathway. A66G variant ( $r$ 1801394) has been reported to affect folate and vitamin B12 status (Hiraoka \& Kagawa, 2017), both of which are essential substrate and cofactor for the successful functioning of the onecarbon metabolism pathway. Based on the interrelated and interconnected reactions, perturbations in this network during embryogenesis may induce serious detrimental effects, including NTD (De Castro et al., 2010). A mouse study has also revealed that hypomorphic mutations in the MTRR gene of either maternal grandparent resulted in NTDs in their grand progeny, regardless of the MTRR genotype in the affected litters, and that this effect was transmitted up to 4 generations (Padmanabhan et al., 2013). This finding implies that a reduction in MTRR gene function has devastating consequences for subsequent generations; hence parental MTRR genotypes are worthwhile to be considered as well.

The objective of this study, if a correlation between MTRR SNP and NTDs is established, may be suggested as a prenatal screening tool for the prevention of 
NTDs. In addition, if future studies can demonstrate that adequate or high folic acid consumption is able to overcome the effects of MTRR A66G variant, the results from this study may serve as a basis for mandatory folic acid fortification in staple foods in Malaysia.

\subsection{MATERIALS AND METHODS}

\subsection{Patient selection / Study subjects}

24 Malaysians were recruited for this study. From here, the 7 patients with sporadic NTDs were grouped into distinct families, comprising of 5 complete trios (mother-father-patient) and 2 single-parent families (mother-patient). There were no descriptions of NTD cases in other family members and none of the patients were the result of consanguineous parents. The patients were presented with spina bifida with neurological deficits, encompassing both spina bifida occulta and aperta. The remaining 5 subjects were healthy individuals unrelated to the patients and were included as normal controls. Blood or saliva samples were collected from participants and all provided written consent and ethical approval was given by the Medical Ethics Committee (MEC) in University of Malaya Medical Centre (UMMC; MEC reference number: 914.5).

\subsection{DNA extraction}

DNA was extracted from either peripheral whole blood samples or saliva of subjects. $5 \mathrm{~mL}$ of the venous blood sample was collected and lysed in a $5 \mathrm{X}$ red cell lysis buffer followed by the addition of proteinase $\mathrm{K}$ in SNET buffer. For saliva collection, Oragene DNA OG-500 and OG-575 kits (Genotek, Canada) were employed. The collection tube was incubated at $50^{\circ} \mathrm{C}$ overnight and subsequent DNA extraction steps were performed according to the manufacturer's protocol. Concentration and purity of extracted DNA were assessed with NanoDrop 8000 (Thermo Scientific, USA) spectrophotometer.

\subsection{Whole exome sequencing and computational analysis}

Five micrograms of each DNA sample was sent to Axeq Technologies for whole exome sequencing (WES). DNA library from the sample was prepared with Illumina TruSeq kit then processed for paired-end sequencing on the Illumina HiSeq 2000 system. Through the integration of tools, the reads were mapped to Genome Reference Consortium Human Build 37 (GRCh37), also known as hg19. MTRR rs1801394 variant was confirmed from each subjects' exome data.
Minor allele frequency was obtained from 4 population databases, namely Genome Aggregation Database (GnomAD), Trans-Omics for Precision Medicine (TOPMED), 1000 Genomes (1000G) and Singapore Genome Variation Project (SGVP). Next, the pathogenicity of the variant was analysed with the following protein function prediction algorithmsSorting Intolerant From Tolerant (SIFT), Protein Variation Effect Analyzer (PROVEAN), Polymorphism Phenotyping V2 (PolyPhen-2) and Simple Modular Architecture Research Tool (SMART).

\subsection{Genotyping and result analysis}

The SNP data from WES were validated through polymerase chain reaction (PCR). PCR reaction was carried out in a final volume of $25 \mu \mathrm{L}$, comprising of 10x DreamTaq ${ }^{\mathrm{TM}}$ Green Buffer (Thermo Fisher Scientific $\left.{ }^{\circledast}\right), 25 \mu \mathrm{M} \mathrm{MgCl}, 20 \mathrm{mg} / \mu \mathrm{L}$ bovine serum albumin (BSA), $10 \mu \mathrm{M}$ dNTP, $10 \mu \mathrm{M}$ of each forward (5'-TGAAGTGATGAGGAGGTTTCTGT-3') and reverse (5'TGTGGTGGTATTAGTGTCCTTT-3') primers, 50 ng genomic DNA, $5 \mu \mathrm{L}$ DreamTaq DNA Polymerase and Milli- ${ }^{\circledast}{ }^{d d H_{2}} \mathrm{O}$ (Merck, Germany).

PCR reactions were performed in a thermal cycler with the following conditions: 4 minutes of initial denaturation at $94^{\circ} \mathrm{C}$, followed by 35 cycles of denaturation at $95^{\circ} \mathrm{C}$, the annealing temperature of $59^{\circ} \mathrm{C}$ and elongation at $72^{\circ} \mathrm{C}$. Each of these 3 steps was performed for 1 minute, followed by a final elongation at $72^{\circ} \mathrm{C}$ for 7 minutes. PCR products were resolved on a $1.2 \%$ agarose gel and sent to First BASE Laboratories Sdn Bhd (Selangor, Malaysia) for Sanger sequencing.

Allelic frequencies and the percentages of subjects carrying variant alleles were calculated based on the number of individuals with homozygous or heterozygous genotypes over the total number of individuals.

\subsection{RESULTS}

\subsection{Details of MTRR SNP investigated in this study}

MTRR variant rs 1801394 is located on chromosome 5, exon2, resulting in a non-synonymous protein change from isoleucine to methionine at position 66 amino acid (Table 1). The missense mutation is a result of a change of $A$ to $G$ nucleotide at position 7870973 . High variant allele frequencies ranging from 0.36400 to 0.46778 (36.40\% to $46.78 \%)$ were detected among populations surveyed in GnomAD, TOPMED and $1000 \mathrm{G}$ databases whereas none were found in the SGVP database. 
The missense mutation is predicted using computational tools to have pathogenic consequences onto MTRR protein function by 2 of the 3 protein algorithms used (Table 2), based on cut-off scores from each computational tool.

\subsubsection{SMART-Ensembl schematic}

Methionine synthase reductase is composed of 698 amino acids, as represented by the horizontal grey line (Figure 1). Domains are presented in boxes, and the pink box shows low complexity region. The dashed red line indicates the position of rs1801394 variant occurred within the flavodoxin domain, which may impact protein function.

\subsection{Exome screening of $M T R R$ and electropherogram} Three genotypes were found in the 24 subjects: $A / A$ (wild type), A/G (heterozygous variant) and $\mathrm{G} / \mathrm{G}$ (homozygous variant). We found that $57 \%$ (4 out of 7 ) of the NTD patients and $83 \%$ (10 out of 12 ) of parents carried variant allele(s) at rs1801394 whereas all 5 controls were wild type for MTRR variant rs1801394 (Table 3), as confirmed from exome data and validated by PCR sequencing (Figure 2 ).

Table 1. Position, effect, and minor allele frequencies of the MTRR variant rs1801394.

\begin{tabular}{llllllll}
\hline Position & Annotation & Base & Amino acid & & \multicolumn{3}{c}{ MAF } \\
\cline { 5 - 7 } & & change & substitution & GnomAD & TOPMED & 1000G & SGVP \\
\hline $\begin{array}{l}\text { Chr5: } \\
7870973\end{array}$ & NM_002454.3 & c.A66G & p.I22M & 0.46778 & 0.43376 & 0.36400 & Not found
\end{tabular}

The position is based on GRCh37.p13. MAF from GnomAD, TOPMED and 1000G databases were calculated from samples sizes of above 2500 each. For SGVP database, the MAF was divided into 3 major population groups in Southeast Asia, M= Malay ( $n=98) ; \mathrm{I}=$ Indian ( $=95) ; C=$ Chinese $(n=99)$.

Table 2. Pathogenicity prediction of MTRR variant rs1801394.

\begin{tabular}{llll}
\hline Prediction Software & SIFT & PROVEAN & PolyPhen-2 \\
\hline Pathogenicity & Damaging & Neutral & Probably damaging \\
(Score) & $(0.002)$ & $(-2.25)$ & $(1.000)$ \\
\hline
\end{tabular}

Cut-off scores for each software: SIFT $=0.050$, PROVEAN $=-2.50$, PolyPhen- $2=0.95$

Table 3. Frequency count of genotypes of rs1801394 in MTRR gene in 19 subjects.

\begin{tabular}{lcccc}
\hline Subjects & \multicolumn{3}{c}{ Genotype } & $\begin{array}{c}\text { Number of subjects with } \\
\text { variant allele(s) }\end{array}$ \\
\cline { 2 - 4 } & AA & AG & GG & $4(57 \%)$ \\
Cases & $3(43 \%)$ & $1(14 \%)$ & $3(43 \%)$ & $10(83 \%)$ \\
Parents & $2(17 \%)$ & $8(66 \%)$ & $2(17 \%)$ & 0 \\
Controls & $5(100 \%)$ & 0 & 0 & Total = 14
\end{tabular}

$A A=$ wild type; $A G$ = heterozygous mutant; Het = homozygous mutant .

\subsection{DISCUSSION}

In this study, we investigated the association of MTRR variant rs1801394 with neural tube defects. 14 out of the 19 Malaysian NTD patients and their parent(s) recruited in this study were confirmed to carry mutated MTRR rs1801394 allele(s), based on whole exome sequencing and subsequent validation by PCR. In each family, at least one member was detected as homozygous or heterozygous variant allele carriers.
Inferring from GnomAD, TOPMED and 1000G databases, the occurrence of this SNP is common with a MAF of 0.36 and above, based on the accepted convention that any mutations recorded in more than $1 \%$ or 0.01 of the general population are considered to be common (Chen et al., 2011), and thus unlikely to bear clinical significance (Kryukov et al., 2007). However, this SNP was not found in the SGVP 


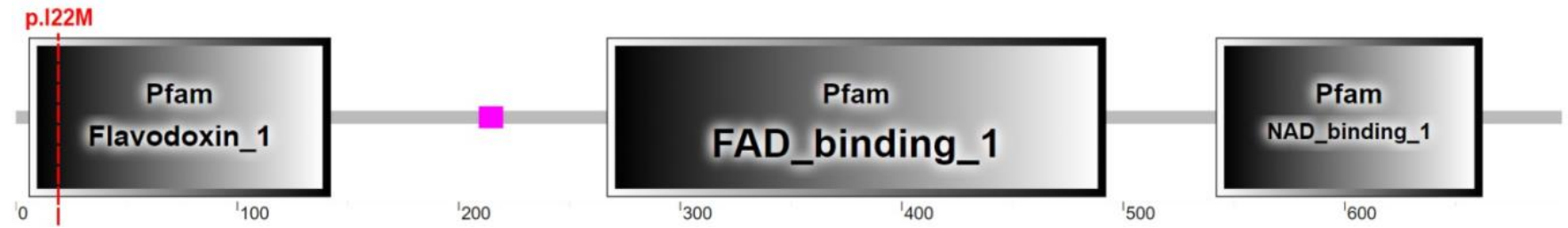

Figure 1. Schematic diagram of MTRR protein domain as predicted by SMART-Ensembl using amino acid sequences
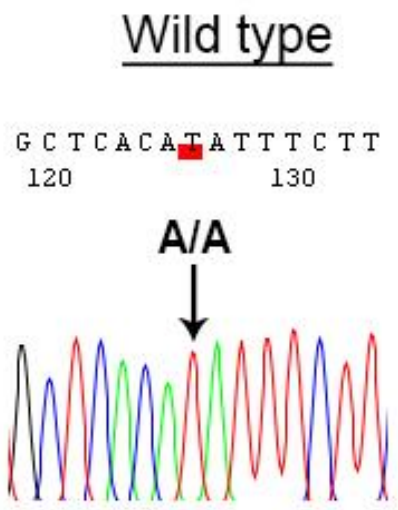
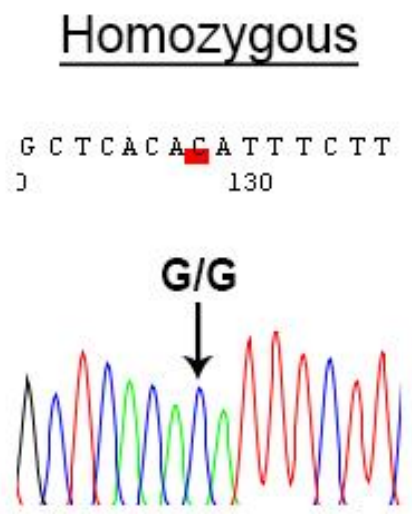

\section{Heterozygous}
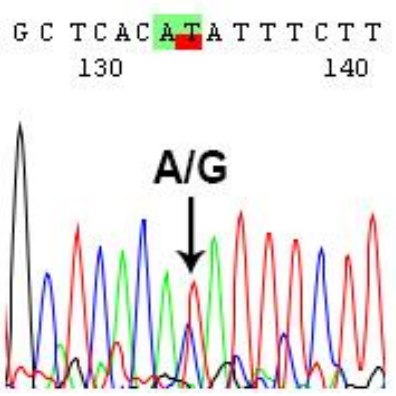

Figure 2. Reverse sequencing data for rs1801394. SNP positions are indicated by arrows.

database, which is interesting as the population group genotyped in SGVP, consisting of 292 Singaporean Malay, Chinese and Indian individuals are a closer representation of the Malaysian patients in our study in terms of genetic ancestries (Teo et al., 2009). This finding is also consistent in our 5 healthy controls, whom comprises of the 3 main races in Malaysia. In the medical genetics field, differences in allele frequency profiles across populations are biologically meaningful (Bomba et al., 2017). Thus, by contrasting the prevalence of MTRR variant rs1801394 in more than half of the patients in our study against the rarity of this polymorphism in its demographic, it suggests that this genomic variation may be a contributing factor towards NTD development among Malaysians. Likewise, the high distribution of this MTRR variant in the unaffected parents hints at an underlying epigenetic inheritance pattern that eventually disrupted the neural tube closure process in their offspring.

Protein function prediction tools provide valuable information on the pathogenicity likelihood of amino acid substitutions induced by the variant. Through evaluating the extent of sequence conservation at the missense site and structural features, the functional impact of the variant is then categorised into threat levels according to each algorithm's predefined threshold scores (Raychaudhuri, 2011). The adoption of 3 prediction tools increased the confidence level of the results and to account for false prediction biases in cases where the score is close to the cut-off value (Dakal et al., 2017). Although PROVEAN estimated that the rs1801394 variant is neutral to the MTRR protein, SIFT and PolyPhen-2 reported the variant as damaging with the score of 1.000 by the latter indicates maximum probability, thus is alarming. Hence, SMARTEnsembl was utilised to shed light on possible functional consequences by studying the affected protein domain.

Methionine synthase reductase protein is expressed as a 698 amino acid long protein with 3 identified domain families- flavodoxin, FAD and NAD. The rs1801394 variant resulting in $122 \mathrm{M}$ substitution affects the flavodoxin domain, which is a cofactor binding site. Biochemical analysis revealed that the methionine amino acid replacement affects interaction and affinity between the protein and its redox partner- methionine synthase (Olteanu et al., 2002). Consequently, decreased reductive methylation and reactivation of methionine synthase results in reduced enzyme efficiency, which impacts the generation of $\mathrm{S}$ adenosylmethionine (SAM) molecule required for the methylation process. It is to be noted, however, that since methionine synthase reactivation is only required 
once in 2000 cycles, the reduction in SAM availability can be tolerated, as corroborated by a study that the demand for SAM during neurulation of embryogenesis is adequate (Leung et al., 2017). Nonetheless, as methionine synthase is the crucial link connecting both folate and methyl cycles, a 3-4 fold compromise on its enzymatic activity may lead to other downstream implications in the overall one-carbon metabolism pathway, which has vital roles in embryogenesis (Xu \& Sinclair, 2015). Although the precise mechanisms have yet been determined, perturbations in this pathway have been linked to NTD development (Li et al., 2016).

Based on the probable pathogenicity of rs1801394 in mediating NTD risk and to overcome the small sample size limitation in this study, we explored available data to estimate the extent of association between this variant and NTD patients in other populations. The MTRR mutant genotype has been suggested to increase the risk of NTD development in Canadians (Wilson et al., 1999), Han Chinese in Tianjin province, China (Fang et al., 2018; Wang et al., 2015), Polish (Pietrzyk et al., 2003), Hispanics (Zhu et al., 2003) and English (Doudney et al., 2009). Conversely, no associations were found in the Dutch (Brouns et al., 2008; van der Linden et al., 2006), Irish (O'Leary et al., $\underline{2005}$ ), Chinese in Liaoning province (Zhang et al., 2019) and central China (Liu et al., 2014). Interestingly, one study reported that the rs1801394 variant protects against NTD and reduces development risk in their Northern United Kingdom cohort (Relton et al., 2004). As with the association analyses on MTHFR C677T where geographical and ethnic differences led to discordant results (Copp et al., 2013), the conflicting reports on MTRR support the multifactorial nature of NTD. This is in line with the hypothesis that polymorphisms in a single one-carbon metabolism gene appear to have but a limited role in overall NTD risk determination and that the sum of gene-gene interaction confers a more pronounced pathological effect compared to individual mutant alone (Relton et al., 2004; Greene et al., 2009).

This study has several limitations. Firstly, it is limited by the lack of detailed clinical data from each patient, which would otherwise help correlate genotype- phenotype relationship with the pathogenesis of this variant. Secondly, a genealogical analysis might uncover distant relationships among NTD patients in our cohort that would aid in understanding the transmission pattern of this variant. Thirdly, a larger dataset would provide a more convincing polymorphism association.

\subsection{CONCLUSIONS}

In summary, we report the association of MTRR variant rs 1801394 in $57 \%$ of NTD patients and a high $83 \%$ prevalence of this variant in NTD parents in Malaysia. In light of the inconsistent prevalence of rs 1801394 variant across worldwide NTD populations, it is thus important to identify population groups whose genetic profiles may predispose them to increased NTD risks. Even though the sample size in this study is small, it provides initial evidence of the need to screen for this polymorphic variant in a larger Malaysian cohort whom may benefit from the prenatal screening of MTRR A66G mutants. Besides, while gaps in knowledge remain between MTRR gene polymorphisms and prevention of NTD through folic acid fortification, the findings of this study are hoped to serve as a basis for public health intervention in Malaysia in the future.

Acknowledgements: Supported by High Impact Research Grant UM.C/625/1/HIR/062 (J-20011-73595) and UM.C/625/1/HIR/148/2 (J-20011-73843) from Universiti Malaya to N.M.A-A., High Impact Research Grant UM.C/625/1/HIR/MOHE/MED/08 (E-000032) from the Ministry of Higher Education Malaysia to N.M.A-A. and A.A.A., Universiti Malaya Research Fund Assistance $(B K P)$ from Universiti Malaya BKS011-2018 to N.M.A-A., A.A.-A. and M.K.T. and Fundamental Research Grant Scheme FRGS/1/2019/SKK08/UM/02/17 (FP090-2019A) from the Ministry of Education Malaysia to N.M.A-A. and A.B.A. We would like to thank all the patients and families for their support.

Author Contributions: N.M.A-A. and A.B.A. conceived and designed the study. A.C.W.T., S.W.M-Z. and N'A.I. performed the experiment, analysed the data and drafted the manuscript. All other authors contributed to manuscript revision, proofread and approved the submitted version.

Conflicts of Interest: The authors declare no conflict of interest.

\section{References}

Amorim, M. R., Lima, M. A. C., Castilla, E. E., \& Orioli, I. M. (2007). Non-Latin European descent could be a requirement for association of NTDs andMTHFR variant $677 \mathrm{C}>\mathrm{T}$ : A meta-analysis. American Journal of Medical Genetics Part A, 143A(15), 1726-1732. https://doi.org/10.1002/ajmg.a.31812

Blencowe, H., Cousens, S., Modell, B., \& Lawn, J. (2010). Folic acid to reduce neonatal mortality from neural tube disorders. International Journal of Epidemiology, 39 Suppl 1(Suppl 1), i110-21. https://doi.org/10.1093/ije/dyq028

Bomba, L., Walter, K., \& Soranzo, N. (2017). The impact of rare and low-frequency genetic variants in common disease. 
Genome Biology, 18(1), 77. https://doi.org/10.1186/s13059-017-1212-4

Brouns, R., Ursem, N., Lindemans, J., Hop, W., Pluijm, S., Steegers, E., \& Steegers-Theunissen, R. (2008). Polymorphisms in genes related to folate and cobalamin metabolism and the associations with complex birth defects. Prenatal Diagnosis, 28(6), 485-493. https://doi.org/10.1002/pd.2006

Chen, H., Hendricks, A. E., Cheng, Y., Cupples, A. L., Dupuis, J., \& Liu, C.-T. (2011). Comparison of statistical approaches to rare variant analysis for quantitative traits. BMC Proceedings, 5 Supp/ 9(Suppl 9), S113. https://doi.org/10.1186/1753-6561-5$\underline{\text { s9-S113 }}$

Copp, A. J., Adzick, N. S., Chitty, L. S., Fletcher, J. M., Holmbeck, G. N., \& Shaw, G. M. (2015). Spina bifida. Nature Reviews Disease Primers, 1(1), 15007. https://doi.org/10.1038/nrdp.2015.7

Copp, A. J., Stanier, P., \& Greene, N. D. E. (2013). Neural tube defects: Recent advances, unsolved questions, and controversies. In The Lancet Neurology (Vol. 12, Issue 8, pp. 799-810). https://doi.org/10.1016/S1474-4422(13)70110-8

Dakal, T. C., Kala, D., Dhiman, G., Yadav, V., Krokhotin, A., \& Dokholyan, N. V. (2017). Predicting the functional consequences of non-synonymous single nucleotide polymorphisms in IL8 gene. Scientific Reports, 7(1), 6525. https://doi.org/10.1038/s41598-017-06575-4

De Castro, S. C. P., Leung, K.-Y., Savery, D., Burren, K., Rozen, R., Copp, A. J., \& Greene, N. D. E. (2010). Neural tube defects induced by folate deficiency in mutant curly tail (Grhl3) embryos are associated with alteration in folate one-carbon metabolism but are unlikely to result from diminished methylation. Birth Defects Research. Part A, Clinical and Molecular Teratology, 88(8), 612-618. https://doi.org/10.1002/bdra.20690

Del Gobbo, G. F., Price, E. M., Hanna, C. W., \& Robinson, W. P. (2018). No evidence for association of MTHFR 677C\&T and 1298A\&C variants with placental DNA methylation. Clinical Epigenetics, 10(1), 34. https://doi.org/10.1186/s13148-0180468-1

Doudney, K., Grinham, J., Whittaker, J., Lynch, S. A., Thompson, D., Moore, G. E., Copp, A. J., Greene, N. D. E., \& Stanier, P. (2009). Evaluation of folate metabolism gene polymorphisms as risk factors for open and closed neural tube defects. American Journal of Medical Genetics Part A, 149A(7), 1585-1589. https://doi.org/10.1002/ajmg.a.32937

Fang, Y., Zhang, R., Zhi, X., Zhao, L., Cao, L., Wang, Y., \& Cai, C. (2018). Association of main folate metabolic pathway gene polymorphisms with neural tube defects in Han population of Northern China. Child's Nervous System, 34(4), 725-729. https://doi.org/10.1007/s00381-018-3730-0

Greene, N. D.E., Stanier, P., \& Copp, A. J. (2009). Genetics of human neural tube defects. Human Molecular Genetics, 18(R2), R113-R129. https://doi.org/10.1093/hmg/ddp347

Greene, Nicholas D E, \& Copp, A. J. (2014). Neural Tube Defects NTDs: Neural tube defects. Annual Review of Neuroscience, 37, 221-242. https://doi.org/10.1146/annurev-neuro-062012-170354

Hayati, A. R., Zainal, A. I., Tan, G. C., Ong, L. C., \& Khoo, T. B. (2008). MTHFR C677T Polymorphism as a Risk Factor of Neural Tube Defects in Malay: A Case Control Study. Medical Journal of Malaysia, 63(5), 379-383.

Hiraoka, M., \& Kagawa, Y. (2017). Genetic polymorphisms and folate status. Congenital Anomalies, 57(5), $142-149$. https://doi.org/10.1111/cga.12232

Kirke, P. N., Mills, J. L., Molloy, A. M., Brody, L. C., O’Leary, V. B., Daly, L., Murray, S., Conley, M., Mayne, P. D., Smith, O., \& Scott, J. M. (2004). Impact of the MTHFR C677T polymorphism on risk of neural tube defects: case-control study. $B M J$ : British Medical Journal, 328(7455), 1535-1536. https://doi.org/10.1136/BMJ.38036.646030.EE

Kondo, A., Matsuo, T., Morota, N., Kondo, A. S., Okai, I., \& Fukuda, H. (2017). Neural tube defects: Risk factors and preventive measures. Congenital Anomalies, 57(5), 150-156. https://doi.org/10.1111/cga.12227

Kryukov, G. V, Pennacchio, L. A., \& Sunyaev, S. R. (2007). Most rare missense alleles are deleterious in humans: implications for complex disease and association studies. American Journal of Human Genetics, 80(4), 727-739. https://doi.org/10.1086/513473

Leung, K.-Y., Pai, Y. J., Chen, Q., Santos, C., Calvani, E., Sudiwala, S., Savery, D., Ralser, M., Gross, S. S., Copp, A. J., \& Greene, N. D. E. (2017). Partitioning of One-Carbon Units in Folate and Methionine Metabolism Is Essential for Neural Tube Closure. Cell Reports, 21(7), 1795-1808. https://doi.org/10.1016/j.celrep.2017.10.072

Li, K., Wahlqvist, M., \& Li, D. (2016). Nutrition, One-Carbon Metabolism and Neural Tube Defects: A Review. Nutrients, 8(11), 741. https://doi.org/10.3390/nu8110741

Liu, J., Qi, J., Yu, X., Zhu, J., Zhang, L., Ning, Q., \& Luo, X. (2014). Investigations of single nucleotide polymorphisms in folate pathway genes in Chinese families with neural tube defects. Journal of the Neurological Sciences, 337(1-2), 61-66. https://doi.org/10.1016/J.JNS.2013.11.017

Mohd-Zin, S. W., Marwan, A. I., Abou Chaar, M. K., Ahmad-Annuar, A., \& Abdul-Aziz, N. M. (2017). Spina Bifida: Pathogenesis, Mechanisms, and Genes in Mice and Humans. Scientifica, 2017, 5364827. https://doi.org/10.1155/2017/5364827

O’Leary, V. B., Mills, J. L., Pangilinan, F., Kirke, P. N., Cox, C., Conley, M., Weiler, A., Peng, K., Shane, B., Scott, J. M., ParleMcDermott, A., Molloy, A. M., \& Brody, L. C. (2005). Analysis of methionine synthase reductase polymorphisms for neural tube defects risk association. Molecular Genetics and Metabolism, 85(3), 220-227.

https://doi.org/10.1016/i.ymgme.2005.02.003

Olteanu, H., Munson, T., \& Banerjee, R. (2002). Differences in the Efficiency of Reductive Activation of Methionine Synthase 
and Exogenous Electron Acceptors between the Common Polymorphic Variants of Human Methionine Synthase Reductase ${ }^{\dagger}$. Biochemistry, 41(45), 13378-13385. https://doi.org/10.1021/bi020536s

Padmanabhan, N., Jia, D., Geary-Joo, C., Wu, X., Ferguson-Smith, A. C., Fung, E., Bieda, M. C., Snyder, F. F., Gravel, R. A., Cross, J. C., \& Watson, E. D. (2013). Mutation in folate metabolism causes epigenetic instability and transgenerational effects on development. Cell, 155(1), 81-93. https://doi.org/10.1016/j.cell.2013.09.002

Pietrzyk, J. J., Bik-Multanowski, M., Sanak, M., \& Twardowska, M. (2003). Polymorphisms of the 5,10methylenetetrahydrofolate and the methionine synthase reductase genes as independent risk factors for spina bifida. Journal of Applied Genetics, 44(1), 111-113. http://www.ncbi.nlm.nih.gov/pubmed/12590188

Raychaudhuri, S. (2011). Mapping rare and common causal alleles for complex human diseases. Cell, 147(1), 57-69. https://doi.org/10.1016/j.cell.2011.09.011

Relton, C., Wilding, C., Pearce, M., Laffling, A., Jonas, P., Lynch, S., Tawn, E., \& Burn, J. (2004). Gene-gene interaction in folaterelated genes and risk of neural tube defects in a UK population. Journal of Medical Genetics, 41(4), 256. https://doi.org/10.1136/JMG.2003.010694

Teo, Y.-Y., Sim, X., Ong, R. T. H., Tan, A. K. S., Chen, J., Tantoso, E., Small, K. S., Ku, C.-S., Lee, E. J. D., Seielstad, M., \& Chia, K.-S. (2009). Singapore Genome Variation Project: a haplotype map of three Southeast Asian populations. Genome Research, 19(11), 2154-2162. https://doi.org/10.1101/gr.095000.109

van der Linden, I. J. M., den Heijer, M., Afman, L. A., Gellekink, H., Vermeulen, S. H. H. M., Kluijtmans, L. A. J., \& Blom, H. J. (2006). The methionine synthase reductase $66 \mathrm{~A}>\mathrm{G}$ polymorphism is a maternal risk factor for spina bifida. Journal of Molecular Medicine, 84(12), 1047-1054. https://doi.org/10.1007/s00109-006-0093-x

van der Put, N., Eskes, T. K., \& Blom, H. J. (1997). Is the common 677C-->T mutation in the methylenetetrahydrofolate reductase gene a risk factor for neural tube defects? A meta-analysis. QJM, 90(2), 111-115. https://doi.org/10.1093/qjmed/90.2.111

Wang, Y., Liu, Y., Ji, W., Qin, H., Wu, H., Xu, D., Tukebai, T., \& Wang, Z. (2015). Analysis of MTR and MTRR Polymorphisms for Neural Tube Defects Risk Association. Medicine, 94(35), e1367. https://doi.org/10.1097/MD.0000000000001367

Wilson, A., Platt, R., Wu, Q., Leclerc, D., Christensen, B., Yang, H., Gravel, R. A., \& Rozen, R. (1999). A Common Variant in Methionine Synthase Reductase Combined with Low Cobalamin (Vitamin B12) Increases Risk for Spina Bifida. Molecular Genetics and Metabolism, 67(4), 317-323. https://doi.org/10.1006/mgme.1999.2879

$\mathrm{Xu}$, J., \& Sinclair, K. D. (2015). One-carbon metabolism and epigenetic regulation of embryo development. Reproduction, Fertility and Development, 27(4), 667. https://doi.org/10.1071/RD14377

Zhang, H., Guo, Y., Gu, H., Wei, X., Ma, W., Liu, D., Yu, K., Luo, W., Ma, L., Liu, Y., Xue, J., Huang, J., Wang, Y., Jia, S., Dong, N., Wang, H., \& Yuan, Z. (2019). TRIM4 is associated with neural tube defects based on genome-wide DNA methylation analysis. Clinical Epigenetics, 11(1), 17. https://doi.org/10.1186/s13148-018-0603-z

Zhang, T., Lou, J., Zhong, R., Wu, J., Zou, L., Sun, Y., Lu, X., Liu, L., Miao, X., \& Xiong, G. (2013). Genetic variants in the folate pathway and the risk of neural tube defects: a meta-analysis of the published literature. PloS One, 8(4), e59570. https://doi.org/10.1371/journal.pone.0059570

Zhu, H., Wicker, N. J., Shaw, G. M., Lammer, E. J., Hendricks, K., Suarez, L., Canfield, M., \& Finnell, R. H. (2003). Homocysteine remethylation enzyme polymorphisms and increased risks for neural tube defects. Molecular Genetics and Metabolism, 78(3), 216-221. https://doi.org/10.1016/S1096-7192(03)00008-8 